\title{
A GENERALIZATION OF THE CAUCHY-SCHWARZ INEQUALITY
}

\section{DAESHIK CHOI}

Abstract. N. Harvey generalizes the Cauchy-Schwarz inequality to an inequality involving four vectors. Here we show a stronger result than the inequality. Moreover we generalize the result to an inequality involving any number of real or complex vectors.

Mathematics subject classification (2010): 26D15.

Keywords and phrases: Cauchy-Schwarz inequality.

\section{REFERENCES}

[1] N. HARVEY, A generalization of the Cauchy-Schwarz inequality involving four vectors, J. Math. Inequal. 9 (2), 2015.

[2] S. DraGomir, A survey on Cauchy-Buniakowsky-Schwartz type discrete inequalities, J. Inequal. Pure \& Appl. Math., 4 (3), 2003. 